\title{
Is there a paradigm shift for GIS data representation and analysis?
}

\author{
Xiaobai A. Yao \\ Department of Geography, University of Georgia, xyao@uga.edu
}

Keywords: Location-based Big Data, GIS, Geospatial Analysis and Modelling

\begin{abstract}
:
In the era of big data, and particularly location-based big data, GIScience is facing significant challenges. The traditional data representational and analytical models have been primarily limited to the view of Newtonian space and time. However, the contemporary enormous amount of location-based social media data and other forms of voluntary geographical data has greatly enhanced the potential to expand the horizon of the field of GIScience by including data that represent more aspects of human activities in the world. For instance, human interactions and information exchange are taking place not only in the physical space but other in virtual spaces, or concurrently in both types of spaces. Similarly, locations may not only exist in the physical space but also in virtual spaces. Social connections may also be traced in either physical or social spaces, or both. Is the shift of ways people interact with each other and with the real world imposing fundamental changes in physical activities in the physical space? If so, how? Ultimately, how can GIS help to organize the data in order to answer new research questions?

This abstract is developed in response to the call for submissions to the research agenda session organized by the commission on geospatial analysis and modeling. Among other important and interesting research directions, I choose to discuss the following topics. I will provide my partial assessment of the current state of knowledge as well as preliminary analysis of associated research questions.
\end{abstract}

1. Revamping the representation framework of current GIS

New representational framework is needed to organize data in spatial, social, and temporal space. Wei and Yao (2018) argued that current GIS representations do not distinguish between spatial location and virtual locations in the virtual space, neither do they account for social associations among people. They proposed an ontological framework that identifies four primary categories in the location-based social media data, namely Agents, Activities, Places, and Social Connections. Such framework is an example of what need to be represented and analysed in future GIS.

2. Representational bias of current location-based social media data

It is widely known that the demography of social media users is not representative of the demography of the general public. However, the location-based social media data are used anyway in many studies regardless of the representative bias. Little has been done to understand the nature of the bias and how the bias impact research findings. There is a dire need for research that can shed light on a better understanding of the bias and on possible responses to the problem.

3. Data fusion

In the era of big data, with a myriad of data sources and data types, how to integrate the heterogeneous data is a challenge task. Yao et al (2019) suggested that developing analytical data fusion approaches is an important research direction for location-based big data.

4. Analytical models for spatio-temporal-social relationships

Traditional GIS analysis and modelling focuses on space and spatial relationships, while sometimes the temporal dimension is also added. However, location-based big data are often acquired from individuals with fine-grained location and time information. Location-based social media data show connections among the individuals. In other words, social connections are embedded in such spatially and temporally informed data. Therefore, it is possible and highly beneficial to explore data in the integrated social-spatial-temporal dimensions. Traditional models were not developed for the high dimensional dynamics. New analytical models are in great demand to analyse the data to discover patterns and relationships in social-temporal-social dimensions. 


\section{References:}

Yao, X. A., Huang, H., Jiang, B., \& Krisp, J. M. (2019). Representation and analytical models for location-based big data. International Journal of Geographical Information Science, 1-7. doi: 10.1080/13658816.2018.1562068

Wei, X. and X. Yao. 2018. A Conceptual Framework for Representation of Location-based Social Media Activities. In Winter, S., Sester, M., Griffin, A. (Eds.), Proceedings 10th International Conference on Geographic Information Science (GIScience 2018), LIPICS Vol. 114. ISBN 978-3-95977-083-5. DOI: 10.4230/LIPIcs.GISCIENCE.2018.62 